\title{
Dynamical mass of a star cluster in M 83: a test of fibre-fed multi-object spectroscopy
}

\author{
S. L. Moll ${ }^{1}$, R. de Grijs ${ }^{1,2}$, P. Anders ${ }^{3}$, P. A. Crowther ${ }^{1}$, S. S. Larsen ${ }^{3}$, L. J. Smith ${ }^{4,5}$, and S. F. Portegies Zwart ${ }^{6,7}$ \\ 1 Department of Physics and Astronomy, University of Sheffield, Sheffield S3 7RH, UK \\ e-mail: s.moll@sheffield.ac.uk; Paul.Crowther@sheffield.ac.uk \\ 2 National Astronomical Observatories, Chinese Academy of Sciences, Beijing 100012, PR China \\ Sterrenkundig Instituut, Universiteit Utrecht, PO Box 80000, 3508 TA Utrecht, The Netherlands \\ ${ }^{4}$ Space Telescope Science Institute and European Space Agency, Baltimore MD 21218, USA \\ Department of Physics \& Astronomy, University College London, London WC1E 6BT, UK \\ 6 Astronomical Institute "Anton Pannekoek", University of Amsterdam, 1098 SJ Amsterdam, The Netherlands \\ 7 Section Computational Science, University of Amsterdam, 1098 SJ Amsterdam, The Netherlands
}

Received 8 May 2008 / Accepted 29 July 2008

ABSTRACT

\begin{abstract}
Aims. We obtained VLT/FLAMES+UVES high-resolution, fibre-fed spectroscopy of five young massive clusters (YMCs) in M 83 (NGC 5236). This forms the basis of a pilot study testing the feasibility of using fibre-fed spectroscopy to measure the velocity dispersions of several clusters simultaneously, in order to determine their dynamical masses. In principle, this reduces the telescope time required to obtain a statistically significant sample of dynamical cluster masses. These can be used to assess the long-term survivability of YMCs by comparing their dynamical and photometric masses, which are necessary to ascertain the potential evolution of YMCs into second-generation globular clusters.

Methods. We adopted two methods for determining the velocity dispersion of the star clusters: cross-correlating the cluster spectrum with the template spectra and minimising a $\chi^{2}$ value between the cluster spectrum and the broadened template spectra. We also considered both red giant and red supergiant template stars. Cluster 805 in M 83 (following the notation of Larsen) was chosen as a control to test the reliability of the results obtained by this observational method, through a comparison with the results obtained from a standard echelle VLT/UVES spectrum obtained by Larsen \& Richtler.

Results. We find no dependence of the velocity dispersions measured for a cluster on the choice of red giant versus red supergiant templates, nor on the method adopted. However, we do find that the standard deviation of the results obtained with only one method may underestimate the true uncertainty. We measure a velocity dispersion of $\sigma_{\text {los }}=10.2 \pm 1.1 \mathrm{~km} \mathrm{~s}^{-1}$ for cluster 805 from our fibrefed spectroscopy. This is in excellent agreement with the velocity dispersion of $\sigma_{\text {los }}=10.6 \pm 1.4 \mathrm{~km} \mathrm{~s}^{-1}$ determined from the standard echelle UVES spectrum of cluster 805. Our FLAMES+UVES velocity dispersion measurement gives $M_{\mathrm{vir}}=(6.6 \pm 1.7) \times 10^{5} M_{\odot}$, consistent with previous results. This value of the virial mass is a factor of $\sim 3$ greater than the cluster's photometric mass, indicating a lack of virial equilibrium. However, based on its effective star formation efficiency, the cluster is likely to virialise, and may survive for a Hubble time, in the absence of external disruptive forces. Unfortunately, our observations of the other M 83 star clusters have insufficient signal-to-noise ratios to determine robust cluster velocity dispersions.

Conclusions. We find that reliable velocity dispersions can be determined from high-resolution, fibre-fed spectroscopy. The advantages of observing several clusters simultaneously outweighs the difficulty of accurate galaxy background subtraction, providing that the targets are chosen to provide sufficient signal-to-noise ratios, and are much brighter than the galaxy background.
\end{abstract}

Key words. galaxies: clusters: general - galaxies: individual: M 83 - galaxies: spiral

\section{Introduction}

The production of luminous, compact star clusters is characteristic of starburst galaxies. However, these clusters have been observed in several different types of galaxy, including normal spirals (see e.g. Larsen 1999, and references therein). These bright clusters are termed Young Massive Clusters (YMCs), and are usually defined as those star clusters that are younger than $\sim 1 \mathrm{Gyr}$, more massive than $\sim 10^{5} M_{\odot}$ and compact, with half-light radii of a few to a few tens of parsecs. The sizes, luminosities and masses of these YMCs are consistent with the properties expected of young globular clusters. This led to the suggestion that YMCs represent globular clusters at an early phase of their evolution (Whitmore et al. 1993;
Schweizer \& Seitzer 1993). This would mean that studying YMCs would be tantamount to observing globular clusters at the epoch of their birth. Since globular clusters are amongst the oldest building blocks in our Galaxy, and are benchmarks for stellar and galactic evolution, understanding their formation and evolution is fundamental in developing our understanding of the fields of large-scale star formation, as well as how galaxies build up and evolve. Determining whether or not YMCs are protoglobular clusters, therefore, is a vital step in gaining insights into these questions.

The simplest criterion that YMCs need to fulfil in order to evolve into globular clusters is that they must be able to survive for a large fraction of a Hubble time. Since a cluster requires sufficient low-mass stars to survive beyond a few Gyr, the 
present-day mass function (PDMF) of a cluster can be used to assess its survivability. It may be possible to get a handle on the PDMF of a cluster by comparing its dynamical mass to its photometric mass, which is the mass predicted by evolutionary synthesis modelling based on the observed luminosity of the cluster and assuming an initial mass function (IMF). If we assume that the dynamical mass of a cluster represents its true mass, any discrepancies between the two masses can be attributed to adopting incorrect assumptions in the photometric mass determination, such as the cluster IMF. Even for a very young cluster, which is not in virial equilibrium, its virial mass can still be used to assess the survivability of the cluster. This is done by by determining how far out of virial equilibrium the cluster is, as quantified by its effective star-formation efficiency (eSFE; Goodwin \& Bastian 2006). Thus, dynamical mass determinations can potentially be used to test the scenario with respect to the long-term survivability of YMCs, (e.g. Ho \& Filippenko 1996a,b; see de Grijs \& Parmentier 2007 for an overview and references therein).

Since the velocity dispersion of a cluster needs to be measured in order to determine the dynamical mass of a cluster (see Sect. 3), high-resolution spectroscopy of the cluster is needed. Large samples of dynamical cluster masses need to be obtained to determine whether YMCs might be proto-globular clusters, and this can be very expensive in terms of telescope time. Observing several clusters at once with a high-resolution, fibre-fed spectrograph limits this cost. However, it is unclear whether a sufficiently accurate galaxy background subtraction can be achieved from only a few fibres placed at a different position to the star cluster. In this paper, we explore the efficiency of this approach, using FLAMES+UVES to observe clusters in M 83. Similar attempts at velocity dispersion measurements have been made with FLAMES+GIRAFFE (e.g. Mieske et al. 2008), which uses an order of magnitude more fibres than FLAMES+UVES. Since large numbers of fibres can be dedicated to galaxy background observations, background subtraction is more straightforward through the use of a master background spectrum. This, however, is at the expensive of at least a factor of two in spectral resolution, and a significantly reduced spectral range. This reduced spectral resolution would, however, not permit the measurement of the small cluster velocity dispersions anticipated for YMCs $\left(\sim 7-15 \mathrm{~km} \mathrm{~s}^{-1}\right)$. Furthermore, Mieske et al. (2008) observed ultra-compact dwarf galaxies (UCDs), rather than clusters. These are much brighter relative to the background, and have a much more uniform background than a spiral like $\mathrm{M} 83$.

M 83, also known as NGC 5236, is a prime example of a nearby spiral galaxy hosting several YMCs (Larsen 2004). At a distance of $4.5 \pm 0.3 \mathrm{Mpc}$ (Thim et al. 2003), with its face-on orientation and multitude of clusters, M 83 is an ideal site to test whether fibre-fed spectroscopy can be used to measure the velocity dispersions of several clusters simultaneously. A further advantage of using M 83 as the testing ground for this method is that the dynamical masses of two of its clusters - clusters 502 and 805 (nomenclature from Larsen 2004) - have already been measured from standard high-resolution echelle spectroscopy (Larsen \& Richtler 2004). Therefore, these clusters can provide a control sample to assess the reliability of the results found from fibre-fed spectroscopy.

M 83 is a metal-rich galaxy, with a central oxygen abundance of $12+\log \mathrm{O} / \mathrm{H}=8.94 \pm 0.09$ (Bresolin et al. 2005), nearly twice the solar value of $12+\log \mathrm{O} / \mathrm{H}=8.69$ (Asplund et al. 2004). The grand-design spiral has a companion, NGC 5253 (Rogstad et al. 1974), with both galaxies hosting intense starburst activity (Calzetti et al. 1999; Tremonti et al. 2001). M 83 has a nuclear starburst and ongoing star formation within its spiral arms (Elmegreen et al. 1998; Harris et al. 2001) and has a star formation rate ${ }^{1}$ of $4 \pm 1 M_{\odot} \mathrm{yr}^{-1}$. The galaxy hosts in excess of 1000 Wolf-Rayet stars (Hadfield et al. 2005).

This paper is structured as follows. In Sect. 2, we present our observations of M 83 and subsequent data reduction, as well as photometry of the clusters and galaxy background regions. In Sect. 3, we determine the velocity dispersion of cluster 805 and compare it with the results obtained from the standard UVES spectrum of Larsen \& Richtler (2004). We discuss the possibility of using fibre-fed spectroscopy to obtain velocity dispersions for other clusters in Sect. 4. Finally, we summarise our findings in Sect. 5.

\section{Observations and data reduction}

We obtained fibre-fed spectroscopy of five clusters and three regions of galaxy background in M 83 with the Fibre Large Array Multi Element Spectrograph (FLAMES) linked with the Ultraviolet and Visual Echelle Spectrograph (UVES) on the Very Large Telescope (VLT) Kueyen Telescope (UT2) in Chile.

\subsection{Imaging of $M 83$}

We retrieved two archival $I$-band images of M 83 taken with the Focal Reducer and low dispersion Spectrograph \#1 (FORS1) on the VLT. These images, taken as part of ESO proposal I.D. 60.A9203(A), were observed on 9th and 11th March 1999 UT for 180 s each. The $I$ filter is centred at $7680 \AA$ with a $1380 \AA$ fullwidth at half-maximum (FWHM), similar to the wavelengths covered by our FLAMES+UVES spectroscopy.

The images were obtained to aid in selecting the clusters and regions of galaxy background to observe with the eight fibres of FLAMES+UVES. The clusters were chosen from the catalogue of Larsen (2004). First, we selected cluster 805 as a 'control' to assess the accuracy of our velocity dispersion measurements, by comparing our results with those of Larsen \& Richtler (2004). We then short-listed bright candidate clusters that were denoted in the catalogue as both 'likely/certain cluster' and having been successfully fitted with the light-profile fitting program ISHAPE (Larsen 1999). The final cluster selection was made on the basis of successfully positioning the fibres on five clusters and three nearby regions of galaxy background with the FLAMES fibrepositioning software, prioritising clusters that were flagged as isolated with smooth galaxy backgrounds in the catalogue.

The final selection comprised the five clusters 347, 645, 747, 805 and 897 (Larsen 2004) and three regions of galaxy background, as indicated in Fig. 1. The galaxy background regions were observed so that they could be used to subtract the galaxy background from the cluster spectra. Therefore, these had a range of intensities, similar to the galaxy backgrounds adjacent to the selected clusters. The "high" fibre was positioned on a region with a strong galaxy background, the 'mid' fibre was positioned on a mid-intensity galaxy background region, while the fibre labelled 'low' was positioned on a region with a faint galaxy background.

The FORS1 images were also used to assess the relative fluxes of the galaxy background regions adjacent to the clusters and within the galaxy background fibres. The images were

\footnotetext{
1 This was determined using the equation of Kennicutt (1998) and adopting the average $\mathrm{H} \alpha$ flux of the galaxy as $7.1 \times 10^{-11} \mathrm{erg} \mathrm{s}^{-1}$ (Kennicutt, priv. comm.) and a representative extinction of $E(B-V) \sim$ 0.5 mag (Hadfield et al. 2005).
} 
Table 1. Table of photometry (taken from Larsen 2004), "background factors" and the average background contribution for the five clusters observed.

\begin{tabular}{lccccccccc}
\hline \hline Cluster & \multicolumn{4}{c}{ Photometry (mag) } & \multicolumn{3}{c}{ Background factors } & Background \\
& $V$ & $U-B$ & $B-V$ & $V-I$ & $814 W$ & Low & Mid & High & contribution $(\%)$ \\
\hline 347 & 18.27 & -0.62 & -0.06 & 0.40 & 19.61 & 1.0 & 0.7 & 0.5 & 79 \\
645 & 18.16 & -0.38 & 0.23 & 0.39 & 19.56 & 1.2 & 0.8 & 0.6 & 89 \\
747 & 17.36 & -0.65 & 0.25 & 0.57 & 19.80 & 1.4 & 1.0 & 0.7 & 76 \\
805 & 16.60 & -0.55 & 0.19 & 0.65 & 17.68 & 1.5 & 1.1 & 0.8 & 62 \\
897 & 16.95 & -0.16 & 0.51 & 0.75 & 19.83 & 1.7 & 1.3 & 0.9 & 86 \\
\hline
\end{tabular}

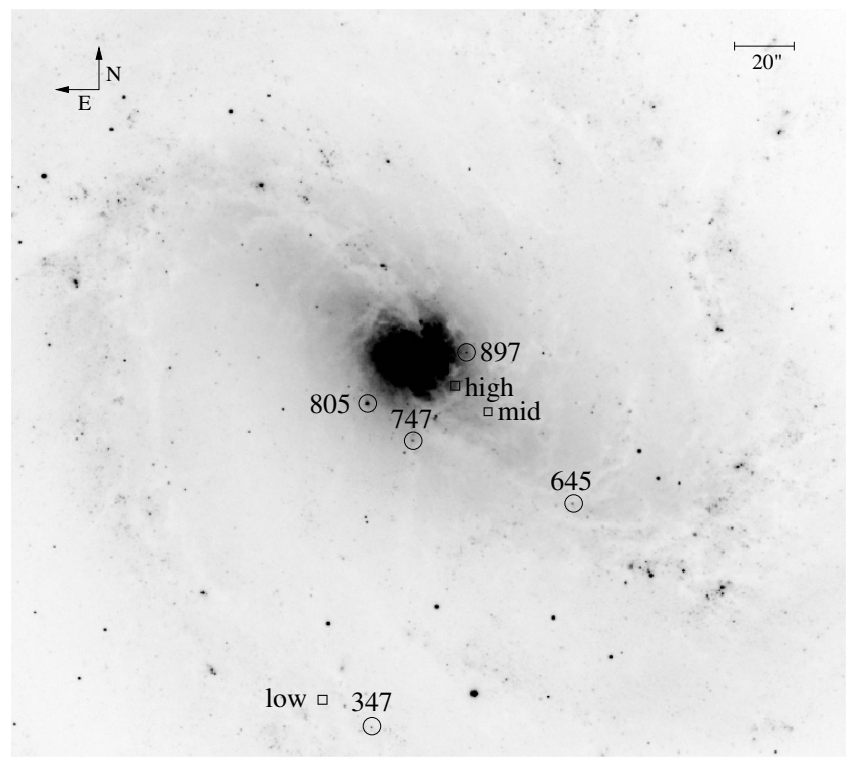

Fig. 1. Archival FORS1 image of M 83 with the five cluster fibre positions and three galaxy background fibre positions indicated.

debiased and flat-fielded with standard calibration frames. We calibrated the astrometry of the images in Starlink's GAIA, using the co-ordinates of the clusters given in the catalogue of Larsen (2004), which were obtained from Hubble Space Telescope (HST) imaging. We carried out our aperture photometry on both images, also using GAIA. We considered the regions of each galaxy background fibre as well as the regions of galaxy background adjacent to each cluster using several small apertures, with radii of both one and two pixels. Taking a mean of these galaxy background values, we computed the 'background factor' for each cluster and galaxy background fibre pair from the relative galaxy background fluxes measured. This background factor is the factor by which the galaxy background flux in the galaxy background fibre needs to be multiplied in order to (approximately) correspond to the galaxy background adjacent to the considered cluster. These background factors are contained in Table 1, along with photometry of the clusters taken from Larsen (2004).

\subsection{Spectroscopy of $M 83$}

We observed the five clusters and three galaxy background regions of M 83 indicated in Fig. 1 over several nights in service mode in May 2006, using FLAMES+UVES under ESO programme ID 077.B 0046(A). The average seeing was $\sim 1$ arcsec. When linked with UVES, FLAMES is a multi-object, highresolution spectrograph $(R=47000)$ with eight fibres of 1 arcsec diameter aperture. In order to position the fibres, each of the eight objects that can be observed at one time must have at least a 10.5 arcsec separation. There are two plates on the fibre positioner, so that the fibres can be positioned on one plate while observations are taking place on the other, thus reducing acquisition time. Each plate has a different spectral response and a slightly different wavelength calibration. Although UVES is a cross-dispersed echelle spectrograph, with both a blue and red arm that can obtain data simultaneously, only the standard red UVES set-ups (centred on 5200, 5800 or $8600 \AA$ ) can be used with FLAMES. This red arm comprises a mosaic of an EEV CCD for the shorter wavelengths and a MIT-LL CCD for the longer wavelengths. We observed for a total exposure time of $22048 \mathrm{~s}$, using cross disperser \#4, centred at $8600 \AA$ with $3121 \mathrm{~mm}^{-1}$, thus obtaining data from $\sim 6670-10425 \AA$. The region $\sim 8545-8645 \AA$ was not observed because it lay within the gap between the two CCDs. ThAr arcs were taken for wavelength calibration. The resolution of the data, as measured from the ThAr arcs, is $\sim 9 \mathrm{~km} \mathrm{~s}^{-1}$. The unbinned data have a pixel scale of 0.182 arcsec pixel ${ }^{-1}$.

Since the spectra of M 83 were faint, we decided that it was more appropriate to reduce the data by hand, rather than to use the pipeline-reduced data. The data were reduced using the software package IRAF, considering the data from each night, and, where relevant, each fibre-positioning plate separately. First, a median bias frame for each night was produced and subtracted from the data. We then divided each data frame by a master slit flat-field for each night. This was produced by taking a median of the nine slit flat-fields that had been taken in three different slit positions each night, rejecting the six faintest frames, and normalising. By rejecting the six faintest frames, the pixels that lay in any of the inter-order gaps in the slit flat-fields were rejected, so that most of the chip was illuminated in the master slit flat-field. All data lay on the illuminated part of this master slit flat-field. The next step was to divide by 'fibre flatfields', which are flat-fields that are obtained through the fibres, and can be used to correct for the relative response of each fibre. We divided by the normalised 2-D all-fibre flat-field. This compared favourably with the results achieved by dividing the extracted data by the fit to the response of extracted odd- and even-fibre flat-fields. All eight fibres of the data were then optimally extracted (Horne 1986), each with an aperture of $1.456 \operatorname{arcsec}(8$ pixels) and wavelength calibrated with the extracted ThAr arcs. We determined the heliocentric radial velocity for each night's data using the Starlink program RV (Wallace \& Clayton 1997) and corrected with these values. The data for each fibre were then co-added, and the orders merged. The 1-D data were then galaxy-background subtracted. This was done by subtracting an average galaxy background spectrum, produced from the two galaxy background fibres with sky factors closest to unity (see Table 1), after multiplication by these sky factors. The relative contribution of the galaxy background to the cluster + galaxy background spectrum is also included in Table 1 .

We measured a recessional velocity of $v_{\mathrm{r}}=500 \pm 4 \mathrm{~km} \mathrm{~s}^{-1}$, after heliocentric radial velocity correction, from the observed 

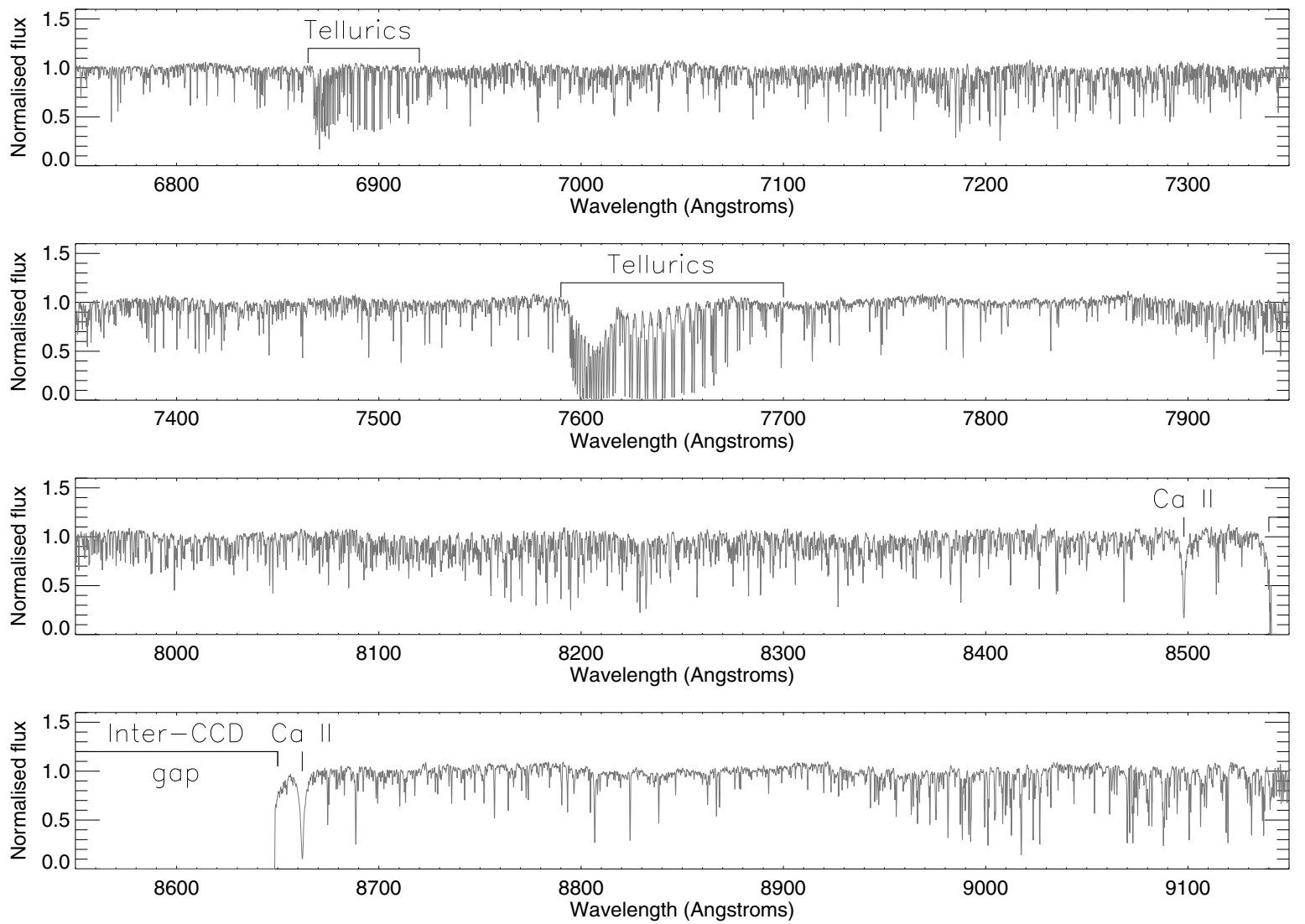

Fig. 2. Pipeline-reduced spectrum of the K1 giant HD 159821, normalised and corrected to rest wavelength, over the wavelength range 6750-9150 ̊. The two lines of the Ca II triplet that are contained in our data ( $8498.02 \AA, 8662.13 \AA)$ are marked. The other line of the Ca II triplet $(8542.09 \AA)$ lies on the gap between the two CCDs, which is also indicated. The major telluric features in this spectral range are also marked.

central wavelengths of the two Ca II triplet lines visible in our data $(8498.02 \AA, 8662.13 \AA$ ) for cluster 805 . These central wavelengths were found by fitting the lines with the ELF (emissionline fitting) routine in Starlink's DIPSO package (Howarth et al. 2004), allowing line width, centre and intensity to vary freely. The recessional velocity implied from the better signal-to-noise ratio $8498 \AA$ line was more heavily weighted, according to the uncertainty in the fitted central wavelength estimated by ELF. This value is in good agreement with other optical measurements: $503 \pm 11 \mathrm{~km} \mathrm{~s}^{-1}$ (de Vaucouleurs et al. 1991), $497 \pm$ $18 \mathrm{~km} \mathrm{~s}^{-1}, 445 \pm 20 \mathrm{~km} \mathrm{~s}^{-1}$ (Fouqué et al. 1992) and $491 \pm$ $30 \mathrm{~km} \mathrm{~s}^{-1}$ (Humason et al. 1956). Since no strong lines, including the $\mathrm{Ca}$ II triplet, could be identified in the spectra of any cluster except cluster 805 , it was not possible to determine individual cluster recessional velocities. Therefore, even though small differences in radial velocity may exist between the clusters, all cluster spectra were velocity corrected by $v_{\mathrm{r}}=500 \mathrm{~km} \mathrm{~s}^{-1}$. The spectra were then normalised, by fitting a polynomial to the continuum across the whole spectrum and dividing by this fit.

\subsection{Spectroscopy of standard stars}

With the setup described above (Sect. 2.2), we also observed a telluric star, CD-32 9927 , and three Galactic red giants HD 159821 (K1 III), HD 123833 (K2 III) and HD 159881 (K5 III). We chose to observe giants rather than supergiants, because these four calibration stars could be observed in only two fields, reducing the overheads of the observations. For each of these standard stars, one sky fibre was placed as close to the object as possible.

We chose to use the pipeline-reduced data for these bright standard stars, since these included a better extraction of the $8662 \AA \mathrm{Ca}$ II triplet lines than the manually-reduced data. This line lies very close to the edge of the MIT-LL CCD for the template stars. Due to their higher recessional velocity, this is not a problem for the M 83 clusters. We sky subtracted the spectra of these bright standards by simply subtracting the relevant sky fibre spectrum. The data were heliocentric radial velocitycorrected using the values computed by RV. Each red giant was then corrected for its recessional velocity using the observed central wavelengths of the two visible $\mathrm{Ca}$ II triplet lines, as measured with ELF. The spectra were then normalised.

An example spectrum of a velocity-corrected, normalised red giant template star, HD 159821 (K1 III) is shown in Fig. 2, for a reduced wavelength coverage. The principal telluric features in this spectral range and the two visible Ca II triplet lines are marked. An example cluster spectrum over suitable spectral regions is shown in Fig. 3, fitted with a red giant template star.

\section{Virial mass}

The virial mass of a cluster, $M_{\mathrm{vir}}$ is given by the equation:

$M_{\mathrm{vir}} \approx \frac{\eta \sigma_{\mathrm{los}}^{2} r_{\mathrm{hl}}}{G}$,

where $\sigma_{\text {los }}$ is the line-of-sight velocity dispersion of the cluster and $r_{\mathrm{hl}}$ is the projected half-light radius of the cluster. This 

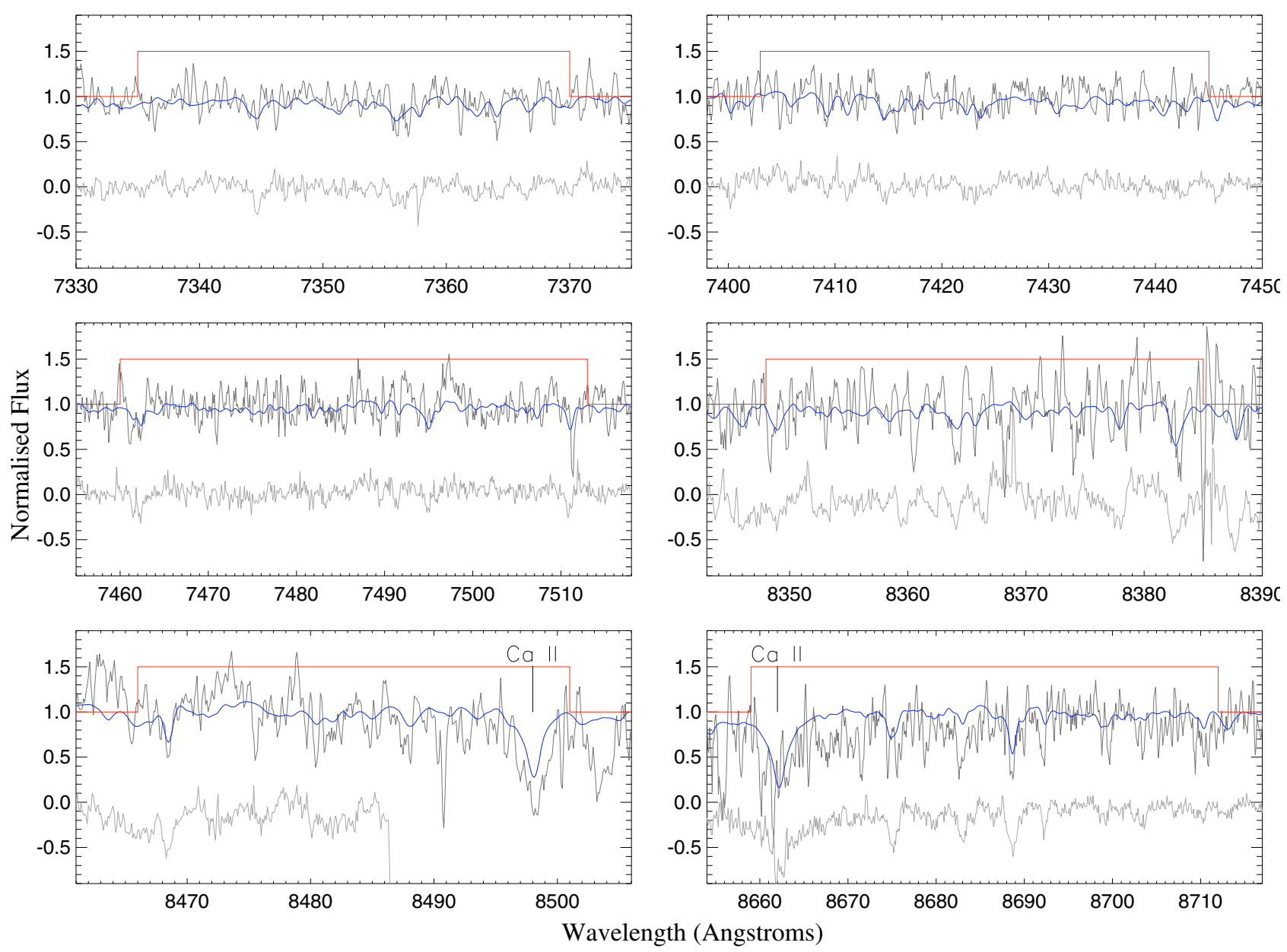

Fig. 3. Best fit of broadening for the K2 III template HD 123833 (blue line) to our normalised FLAMES+UVES cluster 805 spectrum (black line) for the 'Both CaT' case of $10.9 \mathrm{~km} \mathrm{~s}^{-1}$. The spectral regions included in the fit are shown where the red line is at 1.5. Also included in this figure is the standard echelle UVES spectrum of cluster 805 from Larsen \& Richtler (2004) (grey line), offset at -1 . See the online journal for a colour image.

equation follows from the virial theorem, with $\eta=9.75$ for a wide range of light profiles, assuming that the cluster is virialised, gravitationally bound and spherically symmetrical, that the velocity dispersion of the cluster is isotropic and that all of the stars contained in the cluster are single stars of equal mass (Spitzer 1987). However, recent studies have shown that $\eta$ is not a constant, and can vary with time, depending on, for example, the degree of mass segregation and the binary fraction of the cluster (Boily et al. 2005; Fleck et al. 2006; Kouwenhoven \& de Grijs 2008). So that our results are comparable with other dynamical mass determination of YMCs, we adopt $\eta=9.75$, as has been done by other authors.

\subsection{Velocity dispersion}

Clearly, it is necessary to measure the line-of-sight velocity dispersion of the cluster in order to determine the cluster's virial mass. This is achieved by comparing the widths of the Dopplerbroadened lines of the cluster with a template star. The cluster spectrum is compared to either red giant or red supergiant template star spectra, because the lines of these stars are broadened by only a few $\mathrm{km} \mathrm{s}^{-1}$ by macro-turbulence in their atmospheres (Gray \& Toner 1986). It is not appropriate to use earlier type supergiants and main sequence stars, as effects such as rotational broadening, macro-turbulence and micro-turbulence broaden the lines of these stars by amounts substantially greater than the anticipated cluster velocity dispersions. Therefore, only spectral regions redwards of $\sim 5000 \AA$, which are dominated by light arising from cool supergiants of spectral type F-M, should be considered (see e.g. Ho \& Filippenko 1996a). Red supergiant templates are preferable, as young clusters do not contain red giants. However, red giants can be used, although these have lower values of macro-turbulent broadening than more luminous giants. This could mean that comparing the spectrum of a cluster that is dominated by red supergiants with a red giant template spectrum may systematically overestimate the cluster velocity dispersion. However, Larsen et al. (2004) found no systematic differences, within the uncertainties, in the velocity dispersions determined for a cluster when cross-correlating with templates of luminosity classes I, II and III.

Here, we adopted two methods to determine the line-of-sight velocity dispersion. The first involved minimising a $\chi^{2}$ value between the cluster spectrum and broadened template spectra. The second involved cross-correlating the cluster spectrum with each template spectrum. These two methods are discussed in more detail in Sects. 3.1.1 and 3.1.2.

To make a direct comparison between the results that can be obtained with fibre-fed spectroscopy and those from a 
Table 2. Velocity dispersions in $\mathrm{km} \mathrm{s}^{-1}$ determined by comparing our FLAMES+UVES cluster 805 spectrum and the standard UVES cluster 805 spectrum of Larsen \& Richtler (2004) with five red giant (RG) and red supergiant (RSG) template spectra.

\begin{tabular}{|c|c|c|c|c|c|c|c|}
\hline \multirow{3}{*}{ Template } & \multicolumn{5}{|c|}{$\chi^{2}$ minimisation } & \multicolumn{2}{|c|}{ Cross-correlation } \\
\hline & \multicolumn{2}{|c|}{ UVES } & \multicolumn{3}{|c|}{ FLAMES+UVES } & \multirow{2}{*}{$\begin{array}{c}\text { UVES } \\
\text { 'No CaT' }\end{array}$} & \multirow{2}{*}{$\begin{array}{c}\text { FLAMES+UVES } \\
\text { 'No CaT'^ }\end{array}$} \\
\hline & 'No CaT'* & 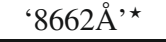 & 'No CaT' * & ' $8662 \AA \AA^{\star}$, & 'Both CaT'* & & \\
\hline K1 III & 10.3 & 10.4 & 9.5 & 9.8 & 9.9 & 9.8 & 9.1 \\
\hline K2 III & 10.8 & 10.9 & 10.2 & 10.6 & 10.9 & 9.8 & 10.0 \\
\hline K5 III & 11.8 & 11.9 & 10.8 & 11.1 & 10.9 & 8.4 & 8.4 \\
\hline $\mathrm{K} 2 \mathrm{Ib}$ & 13.2 & 13.2 & 12.5 & 12.7 & 12.6 & 9.8 & 10.4 \\
\hline K5 Iab & 11.7 & 11.7 & 10.8 & 11.1 & 11.0 & 10.0 & 10.2 \\
\hline Mean of RGs ${ }^{\dagger}$ & $10.9(0.8)$ & $\begin{array}{ll}11.0 & (0.8)\end{array}$ & $10.1(0.7)$ & $\begin{array}{ll}10.5 & (0.7)\end{array}$ & $\begin{array}{ll}10.6 & (0.6)\end{array}$ & $9.3(0.8)$ & $9.2(0.8)$ \\
\hline Mean of RSGs ${ }^{\dagger}$ & $12.4 \quad(1.1)$ & $12.5 \quad(1.0)$ & $11.7(1.2)$ & $11.9 \quad(1.1)$ & $11.8 \quad(1.1)$ & $9.9(0.1)$ & $10.3 \quad(0.1)$ \\
\hline Mean of all ${ }^{\dagger}$ & $11.5(1.1)$ & $11.6 \quad(1.1)$ & $10.8(1.1)$ & $11.1 \quad(1.1)$ & $11.1 \quad(0.9)$ & $9.6 \quad(0.7)$ & $9.6 \quad(0.8)$ \\
\hline
\end{tabular}

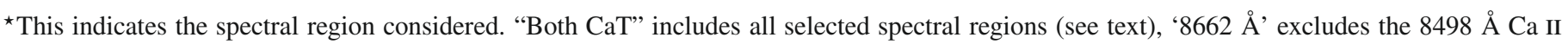
line and "No CaT" excludes both visible Ca II lines.

${ }^{\dagger}$ The standard deviation on each mean is presented in parentheses

standard echelle spectrum, we also determined velocity dispersions using both methods with the non-fibre-fed UVES spectrum of cluster 805 from Larsen \& Richtler (2004). We degraded the UVES spectrum by re-binning the data to the same linear dispersion scale as our own. To consider the effect of red supergiant over red giant templates we also included two red supergiant template stars from the UVES Paranal Observatory Project (POP; Bagnulo et al. 2003): HD 206778 (K2 Ib) and HD 12642 (K5 Iab). These were also re-binned to the same lower linear dispersion scale as our own data.

\subsection{1. $\chi^{2}$ minimisation}

We measured the line-of-sight velocity dispersion of cluster 805 by minimising a $\chi^{2}$ value between the cluster spectrum and each broadened template spectrum in turn. All spectra were normalised and then the continuum was subtracted. The broadening was achieved by convolving a template spectrum with a Gaussian of $\sigma$ equal to the desired velocity broadening. To find the best fit, the $\chi^{2}$-minimisation program considered a range of broadening values, over selected spectral regions. The criteria for selection were that the spectral regions showed visible similarity in absorption lines between the cluster and template spectra and that they did not contain any telluric lines, which could provide an artificial match between the spectra. These spectral regions were: 7335-7370 ̊, 7403-7445 $\AA, 7460-7513 \AA$, 8348-8385 $\AA, 8465-8502 \AA$ and 8659-8712 $\AA$. Rather than considering these small regions of spectra individually, we ran the model over the whole spectrum, but only considered the chosen spectral regions when computing the $\chi^{2}$ value. Non-linear steps of broadening were considered in the $\chi^{2}$-minimisation program, with closer steps as the minimum was approached. An example fit of a broadened template star spectrum to the cluster 805 spectrum over these spectral regions is shown in Fig. 3.

Since the Ca II triplet lines may be saturated in the template spectra, including them in the fit may overestimate the cluster velocity dispersion (e.g. Walcher et al. 2005; Martini \& Ho 2004). However, Mengel et al. (2002) found no disparity between the velocity dispersion results computed by $\chi^{2}$ minimisation for the strongest component of the Ca II triplet and other individual absorption features for clusters in NGC 4038/4039. We decided to consider the potential impact of this effect by excluding these lines from the fit. Therefore, we considered three cases, with each case including slightly different spectral regions in the fit. The first case used the spectral regions described above, including both visible Ca II triplet lines. Thus, it was called 'Both CaT'. We also considered the case where the $8498 \AA \mathrm{Ca}$ II line was masked out of the fit, still including the $8662 \AA \mathrm{Ca}$ II line, since the $8498 \AA$ was not in the UVES spectrum of Larsen \& Richtler (2004). This case was called ' $8662 \AA$ '. In the final case, both $\mathrm{Ca}$ II lines were masked (called 'No CaT'). The results for all of these cases are given in Table 2 .

The results obtained from this method may be sensitive to low signal-to-noise ratios. Taking $\Delta \chi^{2}=1$ as the $1 \sigma$ error (Press et al. 1992), we estimate that the uncertainties on the FLAMES+UVES velocity dispersions are $\sim 2 \mathrm{~km} \mathrm{~s}^{-1}$, while the uncertainties on the UVES velocity dispersions are $\sim 1 \mathrm{~km} \mathrm{~s}^{-1}$.

\subsubsection{Cross-correlation method}

In this second method, we determine the cluster velocity dispersion using the cross-correlation technique of Tonry \& Davis (1979). All spectra were normalised and then the continuum was subtracted. We cross-correlated the cluster spectrum with each template spectrum in turn, over the spectral regions described in Sect. 3.1.1 as 'No CaT', excluding the Ca II triplet lines. We did not consider the other two cases, since broadly consistent results for all three cases were obtained for the $\chi^{2}$ minimisation.

The FWHM of the resulting cross correlation function (CCF) relates to the velocity dispersion of the cluster. This relationship was calibrated by considering the template star used. The template star was broadened and cross-correlated with the original, unbroadened template. By considering several values of broadening, and measuring the FWHM of each CCF, the almost linear relationship between the FWHM of the CCF and broadening was empirically calibrated to an absolute scale, from which the velocity dispersion of the cluster was read. This was repeated for each template, because every star has a different calibration.

This method is less sensitive to spectral-type matching and low signal-to-noise than the $\chi^{2}$ minimisation. However, it suffers from complications associated with the subjectivity of fitting weak, non-Gaussian CCFs. Based on a $5 \%$ error in fitting the FWHMs of the CCFs, each measurement has an uncertainty of $\sim 1 \mathrm{~km} \mathrm{~s}^{-1}$. Note that it is not the intrinsic FWHM that is important, but the relative FWHMs.

\subsection{Comparison of results and techniques}

Firstly, it is apparent from Table 2 that there is no systematic difference in the velocity dispersion measured when comparing 
a cluster spectrum with red giant or red supergiant templates. This is true for both the $\chi^{2}$ minimisation technique and the crosscorrelation. For this reason, we will consider the mean of all five templates when discussing results below.

Secondly, we do not observe a systematic increase in the velocity dispersion measured when the Ca II lines were included in the $\chi^{2}$ minimisation. This may be due to the large wavelength range considered in the fit, thus reducing the weight applied to the strong $\mathrm{Ca}$ II triplet lines. This would also explain the poor fitting of these lines. Due to this similarity in results, we consider the results obtained for all five template stars with the 'No CaT' case of spectral regions for both techniques. The mean and standard deviation of these results give $\sigma_{\text {los }}=10.2 \pm 1.1 \mathrm{~km} \mathrm{~s}^{-1}$.

We find that, within the uncertainties the velocity dispersions found from the FLAMES+UVES data are consistent with those measured from the standard UVES data, for both methods and all spectral regions considered. If we also take a mean of the ten measurements obtained for the two techniques with the 'No CaT' case of spectral regions for the standard UVES data, we find $\sigma_{\text {los }}=10.6 \pm 1.4 \mathrm{~km} \mathrm{~s}^{-1}$, again with the uncertainty representing the standard deviation of the results. This is in excellent agreement with our FLAMES+UVES measurement of $\sigma_{\text {los }}=10.2 \pm 1.1 \mathrm{~km} \mathrm{~s}^{-1}$. Therefore, we conclude that our results agree with those obtained from standard echelle spectroscopy, and that fibre-fed spectroscopy is practicable for the purpose of determining cluster velocity dispersions.

Our FLAMES+UVES velocity dispersion result is also in agreement with the result measured by Larsen \& Richtler (2004) of $\sigma_{\mathrm{los}}=8.1 \pm 0.2 \mathrm{~km} \mathrm{~s}^{-1}$, at the $2 \sigma$ level. This was determined by cross-correlating their UVES spectrum with red supergiant templates. They considered several spectral regions separately:

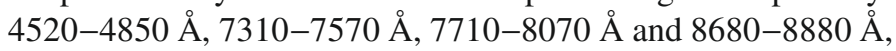
as well as considering two cases including multiple regions: the region 6770-6850 $\AA$ with $6940-7110 \AA$ and the region 6770-6850 A with both 6940-7110 ̊ and 7310-7570 A.

We only obtained results for cluster 805 . The other clusters, which are fainter and have higher relative background contributions, have lower signal-to-noise ratios. We measure a continuum signal-to-noise ratio per resolution element $(\approx 0.1 \AA)$ for cluster 805 of $\sim 7$ at $7000 \AA$ and $\sim 2$ at $9000 \AA$ compared with a signal-to-noise ratio per resolution element for cluster 897 , the next brightest cluster in the $I$-band, of $\sim 6$ at $7000 \AA$ and $\leq 1$ at $9000 \AA$. For these other clusters, the noise is such that no spectral regions could be found for which the absorption lines present in the template star could be identified in the cluster spectrum. Computing the $\chi^{2}$ minimisation over the regions adopted for cluster 805 led to a broadening that was an order of magnitude too large. Cross-correlation over these regions did not produce a peak in the CCF. In the case of cross-correlation, the lack of results cannot be due to differences in radial velocities between these faint clusters. Indeed, had a CCF peak been produced, the radial velocities of these clusters could have been measured from its position.

The rapid decline in the signal-to-noise ratio in the spectra of all clusters, including cluster 805 , redwards of the Ca II meant that we did not include these regions in the velocity dispersion determinations. If the galaxy background was not subtracted from the spectrum of cluster 805, and the sky emission lines were masked out of the fit, the velocity dispersion of the cluster could not be measured. This is probably due to the fact that the lines were significantly diluted by the background. Therefore, this could also not be used to measure the velocity dispersion for any of the other four clusters.

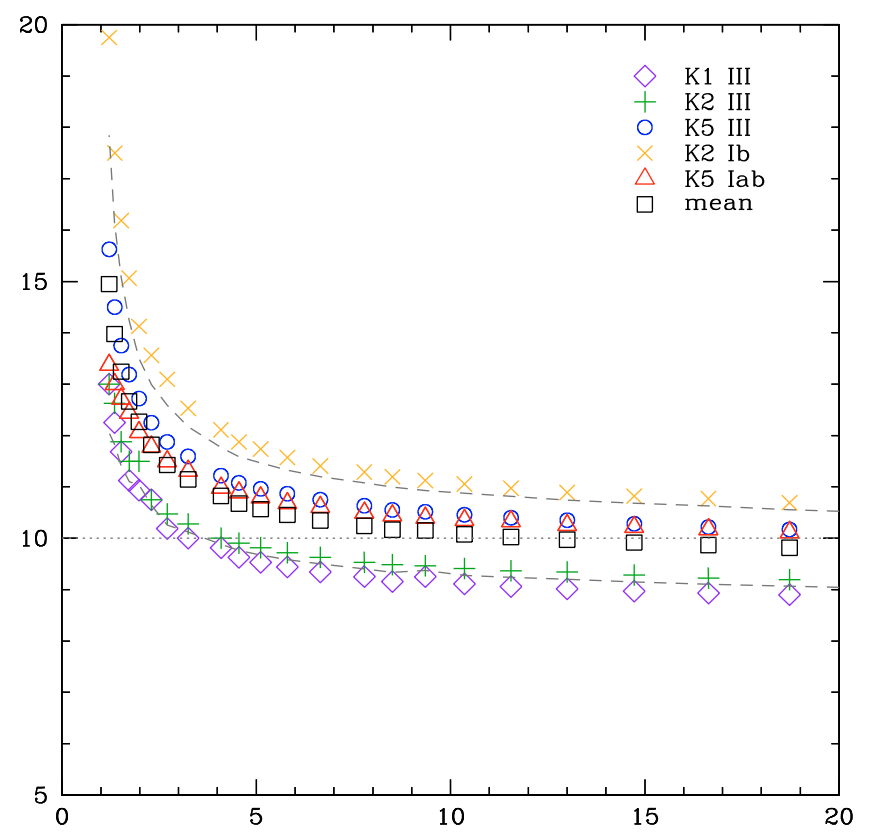

Fig. 4. Plot of velocity dispersion measured from a $\chi^{2}$ minimisation between the five template stars and a simple model cluster spectrum with $\sigma_{\text {los }}=10 \mathrm{~km} \mathrm{~s}^{-1}$ in the 'No CaT' case against the signal-to-noise ratio (SNR) of the model cluster. The mean velocity dispersion measured is also plotted. The dashed lines represent the uncertainty, as measured by the standard deviation if the values, on this mean. The dotted line shows the true velocity dispersion of the model cluster. See the online journal for a colour image.

Clearly, in order to measure reliable star cluster velocity dispersions, good signal-to-noise ratios in the cluster spectra are paramount. We obtained consistent results from cluster 805 with a signal-to-noise ratio of $\gtrsim 3$ in the spectral regions considered, but no results from the data of the other clusters, with lower signal-to-noise ratios. Therefore, a signal-to-noise ratio of $\sim 3$ seems likely to represent a lower limit to the signal-to-noise ratio required to obtain reliable results. To investigate this, we produced a simple model cluster by adding the library K2 Ib and K5 Iab spectra, re-normalising the spectrum and broadening it by $10 \mathrm{~km} \mathrm{~s}^{-1}$. A $\chi^{2}$ minimisation was carried out between this model spectrum and all five template stars for the 'Both CaT' case of spectral region, in an attempt to retrieve this value of the velocity dispersion. The signal-to-noise ratio of the model cluster spectrum was degraded by artificially adding Gaussian noise and the $\chi^{2}$ minimisation was recomputed for the new noisier model spectrum. This was repeated for a range of model cluster spectrum signal-to-noise ratios.

The results are shown in Fig. 4. The mean of the five values measured for each signal-to-noise ratio are also plotted, and an uncertainty on this mean equivalent to the standard deviation is represented by the dashed lines. The measured velocity dispersion increases with decreasing signal-to-noise ratio of the cluster, in agreement with the results found by Martini \& Ho (2004). The plot also shows that, in this case, a cluster signal-to-noise of $\gtrsim 2.1$ is within $20 \%$ of the true broadening, and the lower limit on the signal-to-noise ratio for which the standard deviation of the results describes the true error is just over 3 . The precise signalto-noise ratio at which these minima occur will depend of the cluster and on the nature of the template mismatch, which should be less stark than that illustrated here. This plot indicates that the velocity dispersion measured for cluster 805 is robust, although, as already stated, the standard deviation of the results from the $\chi^{2}$ 
Table 3. Parameters for cluster 805 taken from the literature and determined in this work.

\begin{tabular}{lcl}
\hline \hline Parameter & Value & Reference \\
\hline Distance $(\mathrm{Mpc})$ & $4.5 \pm 0.3$ & Thim et al. (2003) \\
$M_{V}(\mathrm{mag})$ & $-12.2 \pm 0.4$ & Larsen \& Richtler (2004) \\
Age $(\mathrm{Myr})$ & $13 \pm 6$ & Larsen \& Richtler (2004) \\
$M_{\text {phot }}\left(\times 10^{5} M_{\odot}\right)^{\star}$ & $1.9 \pm 1.4$ & Larsen \& Richtler (2004) \\
$r_{\mathrm{hl}}(\mathrm{pc})$ & $2.8 \pm 0.4$ & Larsen \& Richtler (2004) \\
$\sigma_{\text {los }}\left(\mathrm{km} \mathrm{s}^{-1}\right)$ & $10.2 \pm 1.1$ & This work \\
$M_{\mathrm{vir}}\left(\times 10^{5} M_{\odot}\right)$ & $6.6 \pm 1.7$ & This work \\
$L_{V} / M_{\mathrm{vir}}\left(L_{\odot} / M_{\odot}\right)$ & $10 \pm 5$ & This work \\
\hline
\end{tabular}

*Assuming Kroupa (2002) IMF.

minimisation may underestimate the true uncertainty. The larger uncertainty measured from the standard deviation of all five templates with both methods should remain a reliable estimate of uncertainty.

\subsection{Implications for cluster 805}

Adopting the cluster parameters determined for cluster 805 contained in Table 3 , and using the velocity dispersion measured here, we find $M_{\text {vir }}=(6.6 \pm 1.7) \times 10^{5} M_{\odot}$ and a $V$-band luminosity-to-dynamical mass ratio $L_{V} / M_{\text {vir }}=(10 \pm 5) L_{\odot} / M_{\odot}$. These results agree, within the uncertainties, with the values determined by Larsen \& Richtler (2004) of $M_{\mathrm{vir}}=(4.2 \pm$ $0.67) \times 10^{5} M_{\odot}$ and $L_{V} / M_{\text {vir }}=(15 \pm 6) L_{\odot} / M_{\odot}$. Both of these dynamical mass measurements are larger than the photometric mass predicted for a Kroupa (2002) IMF of $M_{\text {phot }}=$ $(1.9 \pm 1.4) \times 10^{5} M_{\odot}$ for the cluster age of $13 \pm 6 \mathrm{Myr}$ (Larsen \& Richtler 2004), indicating that cluster 805 may not be virialised. However, the photometric and dynamical masses agree at just over the $2 \sigma$ level.

Our value of light-to-dynamical-mass ratio suggests that the eSFE of cluster 805 lies between $\sim 30 \%$ and $\sim 50 \%$, favouring the value of $\sim 40 \%$. Goodwin \& Bastian (2006) estimate that a cluster with an eSFE $\sim 30 \%$ will lose $\sim 80 \%$ of its mass within $\sim 40 \mathrm{Myr}$ and disperse. However, a cluster with an eSFE $\gtrsim 40 \%$ will virialise, although it will lose up to $\sim 60 \%$ of it mass, and could survive for a Hubble time in the absence of external disruptive forces.

\section{Discussion: using high-resolution, fibre-fed spectroscopy to determine velocity dispersions}

Using high-resolution, fibre-fed spectroscopy to determine velocity dispersions would have a major impact on producing a large sample of dynamical cluster mass determinations, by making more efficient use of telescope time. By having a large sample of dynamical cluster masses, it should become possible to assess how likely YMCs are to survive for a large fraction of a Hubble time and evolve into globular cluster-type objects.

The primary concern over this method is the question of how successfully the galaxy background could be subtracted from fibre spectroscopy of faint clusters. Of course, sky subtraction is not a problem with bright stars, despite a small amount of flux 'bleeding' from the bright star fibres into the sky fibres. For the fainter objects, galaxy background subtraction is more challenging. Simply scaling and subtracting the galaxy background fibre with the most similar intensity background to the cluster being considered introduces more noise into the spectrum. Taking an average of two or three scaled galaxy background fibres minimises this, although still introduces noise. Subtracting an average of two galaxy background fibres decreases the signalto-noise ratio of the cluster + background spectrum by a factor of $\sim 2.5$ for cluster 805 , the brightest cluster, and a factor of $\sim 4$ for both cluster 347 , the faintest cluster, and cluster 645 , which has the lowest contrast with the galaxy background. It seems that scaling the galaxy background in this way is a reasonable approximation to the local galaxy background. All three galaxy background fibres are very similar, considering the level of noise, in spite of spatial variations in galaxy background, due, for example, to differing stellar populations and extinction. Nevertheless, some sky lines are imperfectly subtracted in the cluster 805 spectrum, although these are within the level of the noise. A reduced $\chi^{2}$ between the low- and mid-background spectra and the scaled, high-background spectrum yields values of $\chi^{2}=0.9$ and $\chi^{2}=0.7$, respectively. While adopting the noise in the spectra as the uncertainty in the fit seems to overestimate the true uncertainty, the relative similarity in the galaxy background spectra, given the large degree of noise, is demonstrated. An alternative method would be to fit the continuum of the scaled, extracted galaxy background fibre and subtract this fit from the cluster spectrum, manually removing the sky lines from the cluster spectrum. However, the extra complications involved in this process were not warranted for our data, since an appropriate galaxy background was achieved from averaged galaxy background fibres.

Also, the signal-to-noise ratio was much higher in the UVES spectrum of Larsen \& Richtler (2004), for which a shorter integration time of $7500 \mathrm{~s}$ was used. This would appear to indicate that long-slit echelle spectroscopy is a more efficient use of telescope time, especially since two clusters could potentially be observed in the same slit. However, finding cases where two clusters can be observed simultaneously can be difficult, due to the relatively short slit length of many echelle sepctrographs (e.g. 11 arcsec for UVES). The signal-to-noise ratio in the galaxysubtracted UVES+FLAMES cluster spectra was substantially lower than anticipated from the ESO exposure time calculator. This predicted a signal-to-noise ratio in excess of 30 at $\sim 8000 \AA$, neglecting the high galaxy background. Even considering this additional noise does not account for the much lower observed cluster signal-to-noise ratios. The most likely explanation for the greatly reduced signal-to-noise ratio is is that the fibres were positioned off-centre. However, this needs to be investigated further.

As discussed in Sect. 3.2, the signal-to-noise ratio of cluster 805 is a good representation of the lowest signal-to-noise ratio required to measure robust velocity dispersions. Therefore, making a conservative assumption that $50 \%$ of the flux of cluster 805 was missed in our spectrum indicates that results could be obtained for a cluster half as bright as cluster 805 (i.e., $m_{I} \approx 16.7 \mathrm{mag}$ ). Approximately 18 of the 159 YMCs in M 83, and 109 of all 1358 YMCs, contained in the catalogue of Larsen (2004) fulfil this criterion.

Another problem with this method, specific to FLAMES+UVES, is the limited choice of spectral region, as dictated by the need to use a standard UVES setup. This meant that one of the strong lines of the Ca II triplet was lost. Furthermore, much of the data in the near-infrared cannot be used to determine velocity dispersions, due to telluric features and the lower CCD response in this spectral region. Clusters also need to be selected carefully, with $M_{\text {vir }} \gtrsim 10^{5} M_{\odot}$ in order to have high enough velocity dispersions to be measured. For a 10-Myr-old cluster, this corresponds to $M_{V}=-11.5 \mathrm{mag}$ 
(STARBURST99; Leitherer et al. 1999), or $m_{V} \sim 16.8$ mag for a distance of $4 \mathrm{Mpc}$ and $E(B-V) \sim 0.1 \mathrm{mag}$.

While the drawbacks of this method must still be borne in mind, we have shown here that reliable velocity dispersions can be measured from fibre-fed spectroscopy. Therefore, this method can potentially be used to obtain a large sample of dynamical masses, which can be used to address the issue of the nature of YMCs as proto-globular clusters.

\section{Summary}

We determined a velocity dispersion of cluster 805 in M 83 of $\sigma_{\text {los }}=10.2 \pm 1.1 \mathrm{~km} \mathrm{~s}^{-1}$ from VLT/FLAMES+UVES spectroscopy. This agrees well with the results obtained here for the standard echelle VLT/UVES spectrum of cluster 805 from Larsen \& Richtler (2004) of $\sigma_{\mathrm{los}}=10.6 \pm 1.4 \mathrm{~km} \mathrm{~s}^{-1}$. This shows that high-resolution, fibre-fed spectroscopy is a practicable method with which to measure the velocity dispersions of several clusters simultaneously. This allows a more efficient use of telescope observing time, providing that sufficiently high signal-to-noise ratios are achieved. Therefore, high-number statistics of YMC dynamical masses, vital for assessing the role of YMCs as proto-globular clusters can be more easily obtained.

We find no systematic differences in the velocity dispersions measured depending on whether red giant or red supergiant templates were considered. Nor do we find any differences in the velocity dispersions determined from the $\chi^{2}$ technique when the $\mathrm{Ca}$ II triplet lines are included in the fit. While the results produced from both a $\chi^{2}$ minimisation and from cross-correlation are consistent, there are indications that adopting the standard deviation of the results determined from only one technique may underestimate the true uncertainties.

Adopting our value of velocity dispersion and all other cluster parameters from Larsen \& Richtler (2004), we measure a virial mass of $M_{\text {vir }}=(6.6 \pm 1.7) \times 10^{5} M_{\odot}$ for cluster 805, somewhat larger than its photometric mass of $M_{\text {phot }}=$ $(1.9 \pm 1.4) \times 10^{5} M_{\odot}$, for a Kroupa (2002) IMF and an age of $13 \pm 6$ Myr. Combining this with the eSFE predictions of Goodwin \& Bastian (2006), we conclude that the cluster will probably virialise and could survive for a Hubble time in the absence of external disruptive forces.

Acknowledgements. We would like to thank Arianne Lançon for providing model red supergiant spectra to help select the spectral regions over which to determine the velocity dispersion. We also appreciate suggestions made by the anonymous referee which have helped to improve this manuscript. Based on observations made with ESO telescopes at the Paranal Observatory with programme ID 077.B-0046(A). The Image Reduction and Analysis Facility IRAF is distributed by the National Optical Astronomy Observatories, which is operated by the Association of Universities for Research in Astronomy, Inc., under cooperative agreement with the U.S. National Science Foundation. SLM acknowledges financial support from STFC.

\section{References}

Asplund, M., Grevesse, N., Sauval, A. J., Allende Prieto, C., \& Kiselman, D. 2004, A\&A, 417, 715

Bagnulo, S., Jehin, E., Ledoux, C., et al. 2003, ESO Messenger, 114, 10

Boily, C. M., Lançon, A., Deiters, S., \& Heggie, D. C. 2005, ApJ, 620, L27

Bresolin, F., Schaerer, D., González Delgado, R. M., \& Stasińska, G. 2005, A\&A, 441, 981

Calzetti, D., Conselice, C. J., Gallagher, J. S., \& Kinney, A. L. 1999, AJ, 118, 797

de Grijs, R., \& Parmentier, G. 2007, ChJA\&A, 7, 155

de Vaucouleurs, G., de Vaucouleurs, A., Corwin Jr., H. G., et al. 1991, Third Reference Catalogue of Bright Galaxies, Version 3.9 (New York: SpringerVerlag)

Elmegreen, D. M., Chromey, F. R., \& Warren, A. R. 1998, AJ, 116, 2834

Fleck, J.-J., Boily, C. M., Lançon, A., \& Deiters, S. 2006, MNRAS, 369, 1392

Fouqué, P., Durand, N., Bottinelli, L., Gouguenheim, L., \& Paturel, G. 1992, Catalogue of Optical Radial Velocities (Lyon: Janvier)

Goodwin, S. P., \& Bastian, N. 2006, MNRAS, 373, 752

Gray, D. F., \& Toner, C. G. 1986, ApJ, 310, 277

Hadfield, L. J., Crowther, P. A., Schild, H., \& Schmutz, W. 2005, A\&A, 439, 265

Harris, J., Calzetti, D., Gallagher III, J. S., Conselice, C. J., \& Smith, A. D. 2001, AJ, 122, 3046

Ho, L. C., \& Filippenko, A. V. 1996a, ApJ, 466, L83

Ho, L. C., \& Filippenko, A. V. 1996b, ApJ, 472, 600

Horne, K. 1986, PASP, 98, 609

Howarth, I. D., Murray, J., Mills, D., \& Berry, D. S. 2004, Starlink User Note 50.24, CCLRC / Rutherford Appleton Laboratory, Particle Physics \& Astronomy Research Council

Humason, M. L., Mayall, N. U., \& Sandage, A. R. 1956, AJ, 61, 97

Kennicutt, R. C. 1998, ARA\&A, 36, 189

Kouwenhoven, M. B. N., \& de Grijs, R. 2008, A\&A, 480, 103

Kroupa, P. 2002, Science, 295, 82

Larsen, S. S. 1999, A\&AS, 139, 393

Larsen, S. S. 2004, A\&A, 416, 537

Larsen, S. S., \& Richtler, T. 2004, A\&A, 427, 495

Larsen, S. S., Brodie, J. P., \& Hunter, D. A. 2004, AJ, 128, 2295

Leitherer, C., Schaerer, C., Goldader, J. D., et al. 1999, ApJS, 123, 3

Martini, P., \& Ho, L. C. 2004, ApJ, 610, 223

Mengel, S., Lehnert, M. D., Thatte, N., \& Genzel, R. 2002, A\&A, 383, 137

Mieske, S., Hilker, M., Jordan, A., et al. 2008, A\&A, 487, 921

Press, W. H., Teukolsky, S. A., Vetterling, W. T., \& Flannery, B. P. 1992, Numerical recipes in FORTRAN. The art of scientific computing, 2nd edn (Cambridge: Cambridge University Press)

Rogstad, D. H., Lockart, I. A., \& Wright, M. C. H. 1974, ApJ, 476, 120

Schweizer, F., \& Seitzer, P. 1993, ApJ, 417, L29

Spitzer, L. 1987, Dynamical Evolution of Globular Clusters (Princeton: Princeton University Press)

Thim, F., Tammann, G. A., Saha, A., et al. 2003, ApJ, 590, 256

Tonry, J., \& Davis, M. 1979, AJ, 84, 1511

Tremonti, C. A., Calzetti, D., Leitherer, C., \& Heckman, T. M. 2001, ApJ, 555, 322

Walcher, C. J., van der Marel, R. P., McLaughlin, D., et al. 2005, ApJ, 618, 237

Wallace, P. T., \& Clayton, C. A. 1997, Starlink User Note 78.9, CCLRC / Rutherford Appleton Laboratory, Particle Physics \& Astronomy Research Council

Whitmore, B. C., Schweizer, F., Leitherer, C., Borne, K., \& Robert, C. 1993, AJ, 106,1354 